\title{
Monitoring perfusion and oxygen saturation in port-wine stains during vascular targeted photodynamic therapy
}

\author{
Defu Chen ${ }^{1,2}$, Ying Wang ${ }^{3}$, Hongyou Zhao ${ }^{1}$, Haixia Qiu ${ }^{3}$, Yongtian Wang ${ }^{2}$, Jian Yang ${ }^{2}$, Ying Gu ${ }^{3,4}$ \\ ${ }^{1}$ Institute of Engineering Medicine, Beijing Institute of Technology, Beijing, China; ${ }^{2}$ Beijing Engineering Research Center of Mixed Reality and \\ Advanced Display, School of Optics and Electronics, Beijing Institute of Technology, Beijing, China; ${ }^{3}$ Department of Laser Medicine, First Medical \\ Center of Chinese PLA General Hospital, Beijing, China; ${ }^{4}$ Precision laser medical diagnosis and treatment Innovation unit, Chinese Academy of \\ Medical Sciences, Beijing, China \\ Contributions: (I) Conception and design: D Chen, Y Gu; (II) Administrative support: Y Wang, YT Wang, J Yang; (III) Provision of study materials or \\ patients: Y Wang, H Zhao, H Qiu, Y Gu; (IV) Collection and assembly of data: D Chen, Y Wang, H Zhao; (V) Data analysis and interpretation: D \\ Chen, Y Wang, H Qiu, J Yang, Y Gu; (VI) Manuscript writing: All authors; (VII) Final approval of manuscript: All authors. \\ Correspondence to: Ying Gu, PhD, MD. Department of Laser Medicine, First Medical Center of Chinese PLA General Hospital, Beijing 100853, \\ China. Email: guyinglaser301@163.com.
}

\begin{abstract}
Background: Vascular targeted photodynamic therapy (V-PDT) is a safe and effective therapeutic modality for port-wine stains (PWS) by targetedly damaging the dilated and malformed blood vessels. This study aims to monitor and quantify the changes in oxygen saturation $\left(\mathrm{StO}_{2}\right)$, blood volume fraction (BVF) and perfusion in PWS lesions before and during V-PDT.
\end{abstract}

Methods: Microvascular parameters (i.e., $\mathrm{StO}_{2}$ and BVF) and skin perfusion were measured noninvasively by using diffuse reflectance spectroscopy (DRS) and laser Doppler imaging (LDI), respectively. The change in $\mathrm{StO}_{2}, \mathrm{BVF}$ and perfusion that occurred in the PWS lesions of 26 patients were monitored and investigated before and during V-PDT in vivo with the systematic administration of the porphyrin-based photosensitizer HiPorfin.

Results: The mean $\mathrm{StO}_{2}(\mathrm{P}<0.05)$, BVF $(\mathrm{P}<0.05)$, and perfusion $(\mathrm{P}<0.001)$ in $\mathrm{PWS}$ lesions of all subjects significantly increased by $6 \%, 34 \%$, and $113 \%$, respectively, 3 min after the initiation of V-PDT. The StO increased first and fluctuated during V-PDT. The overall trend of BVF change was consistent with the perfusion change. The BVF and the perfusion of PWS lesions increased after the initiation of V-PDT, and then gradually decreased.

Conclusions: V-PDT is an effective therapeutic modality in treating PWS. Results showed that LDI and DRS permitted the noninvasive monitoring of the changes in $\mathrm{StO}_{2}, \mathrm{BVF}$, and perfusion in PWS lesions during V-PDT, and these methods can be useful in facilitating our understanding of the basic physiological mechanisms during V-PDT.

Keywords: Port-wine stains (PWS); perfusion; oxygen saturation $\left(\mathrm{StO}_{2}\right)$; blood volume fraction (BVF); vascular targeted photodynamic therapy (V-PDT)

Submitted Apr 09, 2020. Accepted for publication Oct 29, 2020.

doi: $10.21037 / \mathrm{atm}-20-3210$

View this article at: http://dx.doi.org/10.21037/atm-20-3210

\footnotetext{
^ ORCID: 0000-0002-7198-1104.
} 


\section{Introduction}

Port-wine stains (PWS) are congenital and progressive vascular lesions characterized by hyperdilated capillaries predominantly in the superficial dermis and affect $0.3-0.5 \%$ of the population (1). PWS lesions are mostly located on the head and face regions and may cause serious psychological effects $(2,3)$. If untreated, the PWS lesions may progressively darken in appearance with advancing age, and around two-thirds of the lesions develop hypertrophic and nodular components (4). Pulsed dye laser (PDL) therapy is currently the standard treatment modality for PWS. However, only around $10 \%$ of PWS patients achieve complete bleaching after PDL treatment and roughly 20\% of lesions are resistant to PDL (5-7). As an alternative, vascular targeted photodynamic therapy (V-PDT) is considered as a safe and effective treatment for PWS, and has been used successfully to treat PWS in China since the early 1990s (8-11). The side-by-side comparison studies of V-PDT and PDL for the treatment of PWS have demonstrated that V-PDT is at least as effective and safe as PDL and in some cases, superior $(12,13)$. To treat PWS with V-PDT, a photosensitizer is injected intravenously and accumulated in the dilated and malformed blood vessels $(14,15)$. Shortly after the administration of photosensitizer, the PWS lesion is illuminated with a laser light at a specified wavelength, and the photo-excited photosensitizer in the presence of oxygen triggers a cascade of photochemical reactions to generate the intravascular reactive oxygen species (ROS), damaging hyperdilated capillaries in the upper dermis and causing vessel closure without damaging surrounding tissues.

Although V-PDT is a safe and effective therapeutic modality to treat PWS, the treatment outcome varies among different individuals and among multiple sites on the same patient (10). These findings arise from the variations in physician's experience and differences among patients. The objective and accurate assessment of PWS lesions before, during and after V-PDT is desired to mitigate the variability in treatment outcome (16). Furthermore, the use of V-PDT in the treatment of PWS is well established, and its routine use in clinical practice has advanced beyond our understanding of what is happening in PWS lesion during V-PDT treatment $(8,9)$. For this end, several noninvasive optical technologies have been proposed for the pre-, intra-, and post-operative evaluation of PWS, such as laser Doppler imaging (LDI) $(15,17)$, laser speckle imaging $(14,18)$, optical coherence tomography $(19,20)$, photoacoustic imaging (21), spatial frequency domain imaging (22), fluorescence spectroscopy(23) and diffuse reflectance spectroscopy (DRS) $(24,25)$.

The effectiveness of V-PDT is highly dependent on the photosensitizer concentration and the oxygen supply within the target vasculatures, which may be affected by blood perfusion (26). Therefore, monitoring the oxygen during V-PDT may enable prediction of the efficacy of the V-PDT because the cytotoxic effect of V-PDT partly depends on the availability of oxygen. Additionally, the blood perfusion may change due to the vascular damage during V-PDT, and thus these changes may be useful biomarkers for monitoring the microvascular responses to V-PDT. Monitoring the tissue oxygenation and the perfusion during and after PDT may enhance our understanding of the basic physiological mechanisms. LDI is capable of wide-field imaging of skin perfusion, which has been widely used to assess tissue microvascular function $(15,27)$. The perfusion changes in PWS lesion before and during V-PDT can be monitored noninvasively and successfully by using LDI $(15,17)$. The microvascular parameters such as oxygen saturation $\left(\mathrm{StO}_{2}\right)$ and blood volume fraction (BVF) of PWS lesions can be determined quantitatively by fitting the DRS by utilizing the established Farrell's equation with the vessel packaging correction factor $(24,25)$. The feasibility of using DRS for assessing the $\mathrm{StO}_{2}$ and BVF of PWS before and during V-PDT has been validated $(24,25)$. Therefore, monitoring the microvascular parameters (i.e., $\mathrm{StO}_{2}$ and $\mathrm{BVF}$ ) and perfusion in PWS before and during V-PDT by using DRS and LDI may provide a deeper understanding of the response of microcirculation and oxygenation in PWS lesions to V-PDT, and have the potential to guide clinical V-PDT $(14,18)$.

In this study, the changes in the microvascular parameters (i.e., $\mathrm{StO}_{2}$ and $\mathrm{BVF}$ ) and perfusion that occurred in PWS lesions of 26 patients during V-PDT in vivo with the systematic administration of the porphyrin-based photosensitizer HiPorfin were noninvasively measured for the first time by utilizing a customized DRS detection system and a LDI system, respectively. The changes in the $\mathrm{StO}_{2}, \mathrm{BVF}$, and perfusion of PWS lesions before and during V-PDT were monitored and investigated.

We present the following article in accordance with the STROBE reporting checklist (available at http://dx.doi. org/10.21037/atm-20-3210). 
Table 1 Details of the PWS patients in this study

\begin{tabular}{|c|c|c|c|c|c|c|c|c|}
\hline Case & $\begin{array}{c}\text { Age } \\
\text { (years) }\end{array}$ & Sex & PWS type & $\begin{array}{l}\text { Location of scan- } \\
\text { ning }\end{array}$ & Treatment history & \multicolumn{2}{|c|}{ V-PDT dose } & $\begin{array}{l}\text { Rate of color } \\
\text { bleaching (\%) }\end{array}$ \\
\hline 2 & 14 & Male & Purple with proliferation & Cheek & V-PDT, PDL & 100 & 20 & 25 \\
\hline 5 & 10 & Female & Purple & Cheek & PDL & 100 & 20 & 50 \\
\hline 6 & 14 & Female & Purple with proliferation & Lateral cheek & None & 100 & 15 & 25 \\
\hline 7 & 12 & Female & Purple & Cheek & None & 100 & 20 & 50 \\
\hline 11 & 4 & Female & Purple & Cheek & None & 80 & 15 & 25 \\
\hline 12 & 4 & Male & Purple & Lateral cheek & None & 80 & 20 & $75-100$ \\
\hline 13 & 8 & Female & Purple & Cheek & None & 80 & 20 & $75-100$ \\
\hline 14 & 20 & Male & Purple & lower jaw & V-PDT & 100 & 20 & 50 \\
\hline 15 & 19 & Male & Purple & Cheek & V-PDT, PDL & 100 & 25 & 25 \\
\hline 16 & 20 & Male & Purple & Cheek & PDL & 100 & 20 & 50 \\
\hline 17 & 13 & Female & Purple & Cheek & PDL & 100 & 15 & $50-100$ \\
\hline 23 & 11 & Male & Red & Temple & None & 100 & 20 & 25 \\
\hline 24 & 24 & Female & Purple with proliferation & Cheek & V-PDT, PDL & 100 & 15 & LTF \\
\hline 25 & 24 & Female & Purple & Cheek & V-PDT & 100 & 20 & 25 \\
\hline 26 & 9 & Male & Purple & Upper eyelid & None & 100 & 15 & 50 \\
\hline
\end{tabular}

PWS, port-wine stains; V-PDT, vascular targeted photodynamic therapy; PDL, pulsed dye laser; LTF, loss to follow up.

\section{Methods}

\section{Study population}

A total of 26 Chinese patients with PWS aged from 4 to 58 years old at the Department of Laser Medicine in First Medical Center of Chinese PLA General Hospital between August 2014 and August 2016 were recruited in this study
(Table 1). The exclusion criteria were as follows: (I) patients with other vascular malformations, (II) patients with vesselrelated syndromes, (III) patients with other skin diseases. All procedures performed in this study involving human participants were in accordance with the Declaration of Helsinki (as revised in 2013). The study was approved by the Ethics Committee of Chinese PLA General Hospital 
(No. 20100404). Prior to the experiments, informed consent was taken from all the PWS patients (or guardians).

\section{LDI system}

Skin perfusion was determined using a commercially available LDI system (moorLDLS2-IR, Moor instrument), which was used previously to monitor perfusion changes in PWS during V-PDT $(15,17)$. Briefly, the LDI system scans a line of laser light $(\sim 785 \mathrm{~nm})$ over the region of interest that the blood perfusion image was built up line by line. The distance between the head of the LDI scanner and the skin being measured was fixed at $15 \mathrm{~cm}$. The images of a size $15 \mathrm{~cm} \times 12 \mathrm{~cm}$ with the resolution of $256 \times 256$ pixels were collected.

\section{DRS system}

The details of the DRS system and the analyzing model used could be found elsewhere (24,25). Briefly, a customized quadfurcated assembly, which consisted of four optical Ultra-Violet/Visible fibers arranged in a linear array, was used to deliver the input and output light. The core diameter of each fiber was $200 \mu \mathrm{m}$, and the center-tocenter separation between adjacent fibers was $260 \mu \mathrm{m}$. A 20-W tungsten halogen lamp with a spectrum ranging from 360 to 2,000 nm (HL-2000-HP; Ocean Optics, Inc.) was connected to one of the fibers, and the white light was delivered to the skin being investigated. The remitted light was collected and fed back to the miniature spectrometer (QE65000; Ocean Optics, Inc.) through another fiber. The source-detector fiberoptic distance $(520 \mu \mathrm{m})$ was chosen in accordance with the previous studies $(24,28)$. The backscattered spectra ranging from 450 to $800 \mathrm{~nm}$ were recorded for data processing. The $\mathrm{StO}_{2}$ and the $\mathrm{BVF}$ of PWS lesions can be obtained by fitting the DRS.

\section{Study protocols}

The PWS lesions of 26 patients received V-PDT. Before $\mathrm{V}$-PDT, all the PWS lesions and contralateral normal skins were measured by utilizing LDI and DRS, respectively. Then, the surrounding skin not receiving therapy was covered with a light-proof soft cloth to avoid exposure to the treatment light during the V-PDT. A diode-pumped all-solid-state frequency-doubled Nd: YAG laser (Beijing Newraysing Laser Tech Co., Ltd., Beijing, China) with a central wavelength of $532 \mathrm{~nm}$ transformed by a $600 \mu \mathrm{m}$ flat- cut fiber combined with an optical beam shaping system was served as the treatment light source for V-PDT.

Afterward, all patients with PWS were injected intravenously with the domestically produced porphyrinbased photosensitizer HiPorfin (Chongqing Huading Modern Biopharmaceutics Co., Ltd., Chongqing, China) with a dose of 2-3 $\mathrm{mg}$ (HiPorfin) $/ \mathrm{kg}$ body weight. Immediately after the administration of HiPorfin, the PWS lesions were illuminated with the laser light with a wavelength of $532 \mathrm{~nm}$ at a power density of $80-100 \mathrm{~mW} / \mathrm{cm}^{2}$ and an energy density of $72-150 \mathrm{~J} / \mathrm{cm}^{2}$. The irradiation time was ranging between 15 and $25 \mathrm{~min}$ (Table 1). The changes in the microvascular parameters (i.e., $\mathrm{StO}_{2}$ and BVF) and the perfusion in PWS were measured at 3, 5, 7, 10, 15, 20, and 25 min during V-PDT by using LDI and DRS, respectively. The temperature was monitored at each time point by using an infrared thermometer (ST631, Sentry Optronics Corp., Taipei). All the measurements were performed in a temperature-controlled room $\left(24 \pm 1^{\circ} \mathrm{C}\right)$. Before the measurement, all the participants had an acclimatization period. During the measurement, they were asked to remain still to minimize the external influence.

\section{Treatment outcome evaluation}

The digital photographs of the PWS lesions of all patients were collected before V-PDT and at follow-up. All the photographs were assessed by an independent well-trained clinician by using a five-level grading scale (14): 0 , no significant change; $25 \%$, minimal lightening; $50 \%$, obvious lightening; $75 \%$, slight residual color; and 100\%, appears as normal skin.

\section{Data analysis}

The perfusion data were analyzed using the built-in processing software (moorLDLS laser Doppler line scanner research version 2.2, Moor Instruments Ltd.). The paired Student's $t$-test was used to compare the differences, and $\mathrm{P}$ values $<0.05$ were considered statistically significant. Data were graphed using the OriginPro 8.0 software and presented as means with the standard deviation (SD).

\section{Results}

\section{Blood perfusion, BVF and $\mathrm{StO}_{2}$ in PWS before $\mathrm{V}-\mathrm{PDT}$}

Figure 1 shows the representative skin perfusion images and 

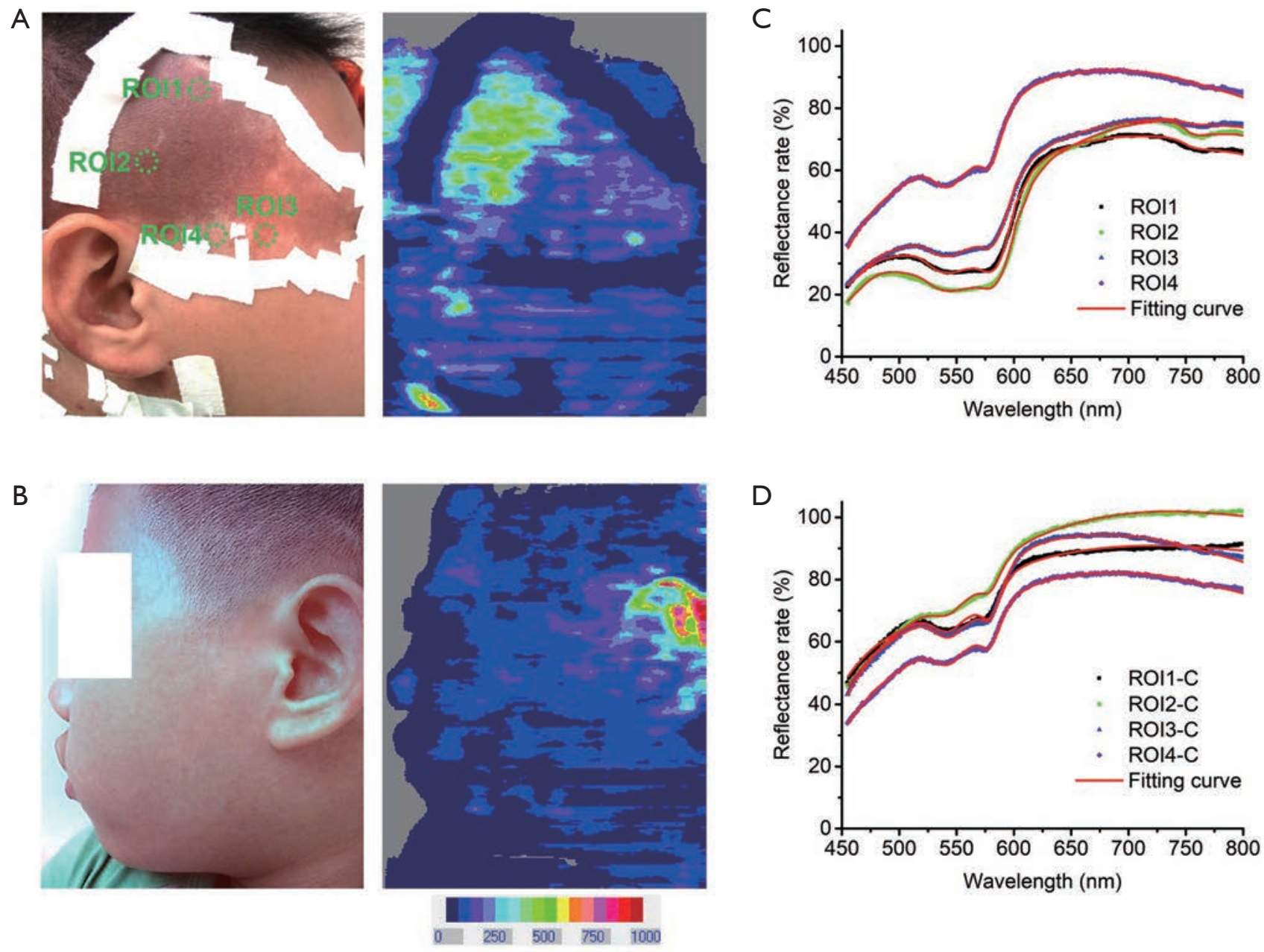

$\mathrm{E}$

$\mathrm{F}$

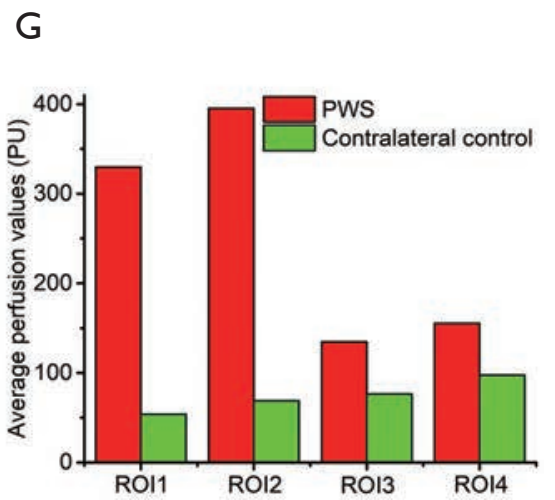

Figure 1 Representative $\mathrm{StO}_{2}$, BVF and perfusion of PWS lesion and the contralateral control site of an 11-year-old male PWS patient before V-PDT (Case 23). (A) Digital image and correlated perfusion image of PWS lesion. (B) Digital image and correlated perfusion image of contralateral control site. (C,D) Diffuse reflectance spectra in PWS lesion and the contralateral control site. The mean (E) $\mathrm{StO}_{2}$, (F) BVF and (G) perfusion taken from ROI1, ROI2, ROI3 and ROI4 in PWS lesion and the contralateral control site. PWS, port-wine stains; $\mathrm{StO}_{2}$, oxygen saturation; BVF, blood volume fraction. 

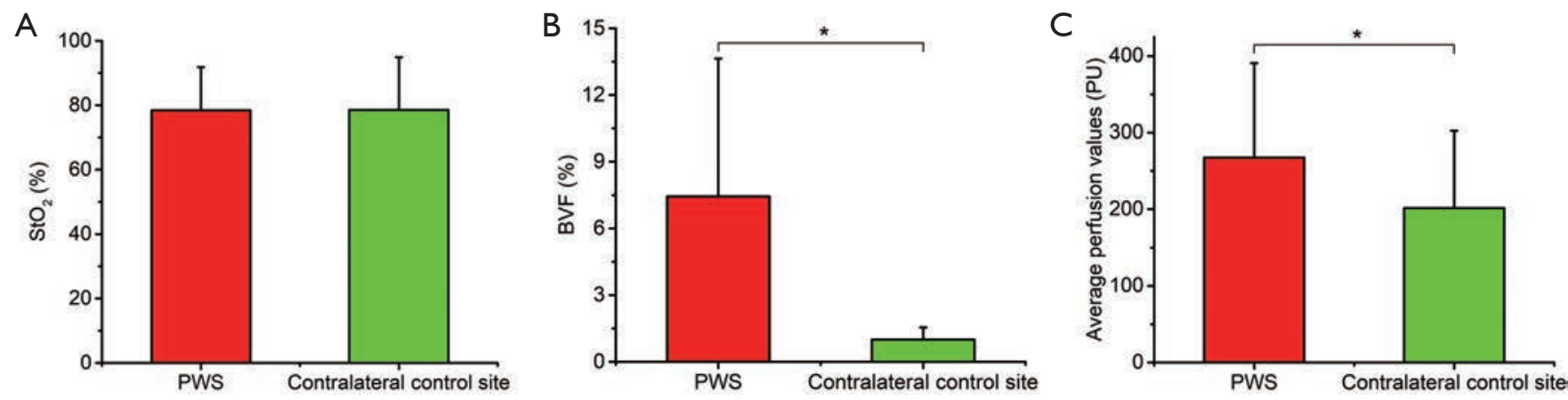

Figure $2 \mathrm{StO}_{2}$, BVF and perfusion of PWS lesions and their contralateral control sites of all patients before V-PDT. (A) StO ${ }_{2}$, (B) BVF and (C) perfusion in the PWS lesions and their contralateral control sites. The error bars represent the SD of the data. * $\mathrm{P}<0.001$. PWS, port-wine stains; $\mathrm{StO}_{2}$, oxygen saturation; BVF, blood volume fraction.

raw diffuse reflectance spectra obtained using by LDI and DRS, respectively, of an 11-year-old male patient with PWS (case No. 23 in Table 1). The perfusion of PWS (Figure 1A) was higher than that of the contralateral control site (Figure 1B). Diffuse reflectance spectra from both PWS and contralateral normal skin clearly showed hemoglobin and oxyhemoglobin absorption bands between 500 and $600 \mathrm{~nm}$. PWS lesions (ROI1, ROI2, ROI3) demonstrated stronger absorption than the contralateral normal skin over the whole spectral range, which may be due to the abundant blood vessels in PWS lesions (Figure 1C,D). The $\mathrm{StO}_{2}$ in the PWS lesion were close to that of the contralateral control sites (Figure 1E). The mean BVF and perfusion obtained from ROI1, ROI2, and ROI3 in the PWS lesion were significantly higher than that of the contralateral heathy control sites (Figure 1F,G). Furthermore, even in the same PWS lesion, the $\mathrm{StO}_{2}, \mathrm{BVF}$, and perfusion were highly heterogeneous.

As shown in Figure 2, among the 26 patients with PWS, no significant difference $(\mathrm{P}>0.05)$ was observed in the $\mathrm{StO}_{2}$ between the PWS lesions and their contralateral control sites before V-PDT. The BVF and perfusion of PWS lesions were significantly greater $(\mathrm{P}<0.001)$ than those of their contralateral control sites. Majority of the PWS lesions (92\%) showed increased BVF compared with the contralateral control sites, whereas $70 \%$ of the PWS lesions showed increased perfusion. Furthermore, the inter- and the intra-patient heterogeneity of $\mathrm{StO}_{2}, \mathrm{BVF}$, and perfusion in PWS lesions were substantial in all subjects.

\section{Changes in blood perfusion, $\mathrm{BVF}$ and $\mathrm{StO}_{2}$ in $\mathrm{PWS}$ during V-PDT}

Figure 3 shows the representative changes in $\mathrm{StO}_{2}, \mathrm{BVF}$, and perfusion of PWS with time during V-PDT (case no. 10 in Table 1). As shown in Figure $3 A$, the PWS lesion became darker and showed mild edema immediately after V-PDT. The skin perfusion of the PWS lesion was heterogeneous and relatively low before V-PDT, and the overall level of perfusion remarkably increased after the initiation of V-PDT and then gradually decreased (Figure 3B,E). Similar to perfusion, BVF increased first and then decreased gradually, although small fluctuations existed (Figure 3D). Furthermore, the changes in BVF and perfusion in the three ROIs within the same PWS lesion showed that the extents of increase and decrease in the perfusion differed from area to area but had the same trend. Unlike perfusion and BVF, $\mathrm{StO}_{2}$ increased slightly and then fluctuated (Figure 3C).

Figure 4 shows the changes in $\mathrm{StO}_{2}, \mathrm{BVF}$, and perfusion in PWS lesions as a function of treatment time during V-PDT. Table 2 shows the summary of changes in the $\mathrm{StO}_{2}$, BVF, and perfusion in all 26 patients during V-PDT. For all the 26 patients, the mean $\mathrm{StO}_{2}(\mathrm{P}<0.05), \mathrm{BVF}(\mathrm{P}<0.05)$, and perfusion $(\mathrm{P}<0.001)$ significantly increased 3 min after the initiation of V-PDT (Figure 4). The mean $\mathrm{StO}_{2}, \mathrm{BVF}$, and perfusion increased by $6 \%, 34 \%$, and $113 \%$, respectively, 3 min after the initiation of V-PDT (Figure 4). $\mathrm{StO}_{2}$ increased first and then fluctuated. The overall trend of BVF change in PWS was consistent with perfusion change, and the BVF and the perfusion of PWS lesions increased after the initiation of V-PDT and then gradually decreased. The infrared thermometer revealed that the temperature of the PWS lesions being treated increased to about $39-42{ }^{\circ} \mathrm{C}$ during V-PDT.

Immediately after V-PDT, most of the PWS lesions became darker or purple and showed mild edema. No obvious side effects (e.g., blistering, erythema, or scar) were 


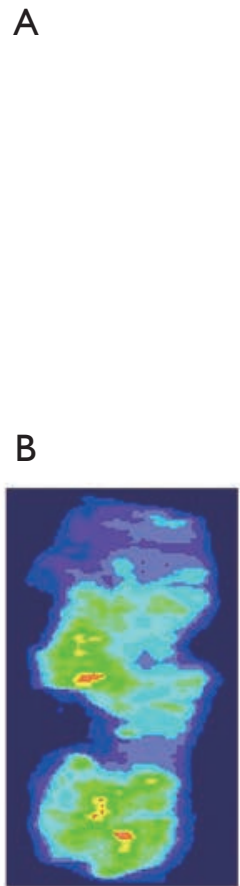

Immediately before
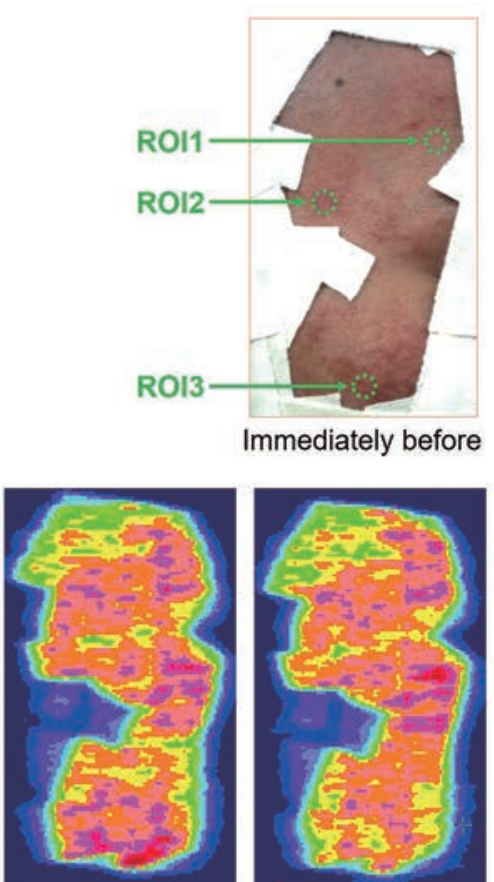

$3 \mathrm{~min}$

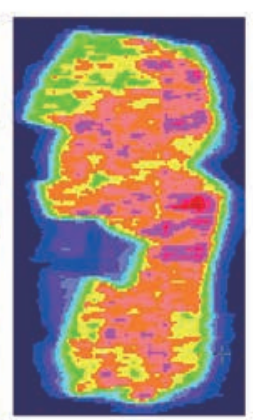

$5 \mathrm{~min}$
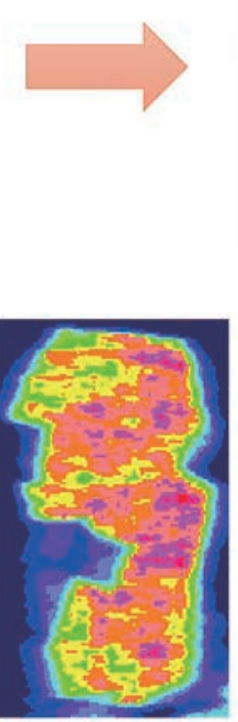

$7 \mathrm{~min}$

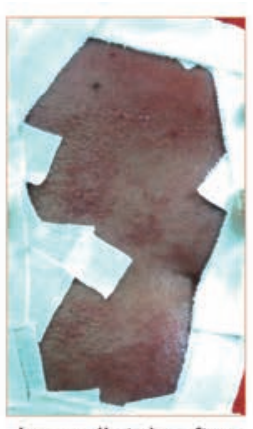

Immediately after

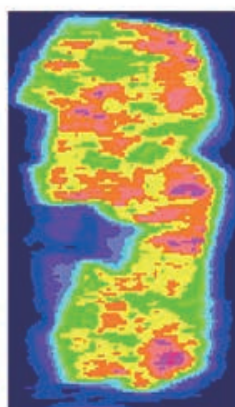

$10 \mathrm{~min}$

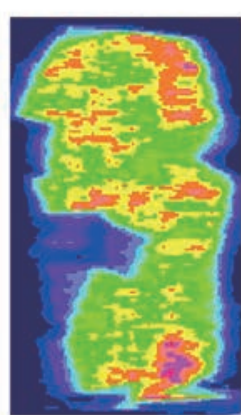

$15 \mathrm{~min}$

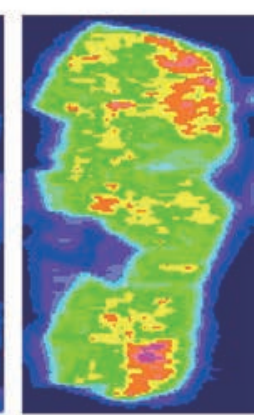

$20 \mathrm{~min}$
C

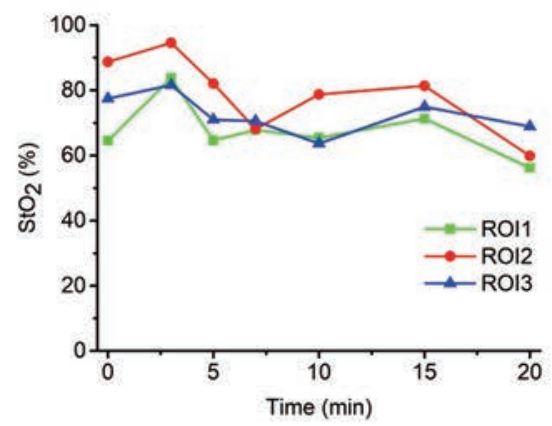

D

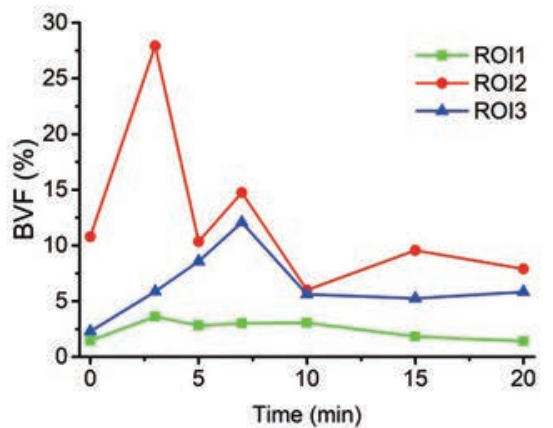

E

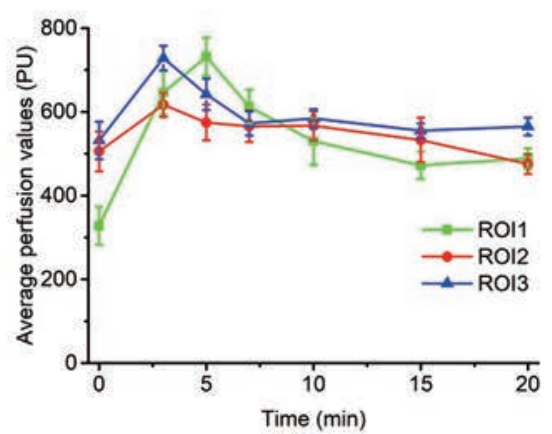

Figure 3 Representative $\mathrm{StO}_{2}$, BVF and perfusion changes in the PWS lesion of a 25-year-old male PWS patient during V-PDT (Case 10). (A) Digital images of PWS lesion immediately before and after V-PDT; (B) the perfusion dynamics during V-PDT. The mean (C) StO 2 , (D) $\mathrm{BVF}$ and (E) perfusion as a function of treatment time taken from marked ROI1, ROI2, and ROI3. PWS, port-wine stains; StO ${ }_{2}$, oxygen saturation; BVF, blood volume fraction.

observed during this study. At the follow-up after treatment, 23 patients had varying degrees of fading (Table 1 lists the rates of color bleaching for the 23 patients, and other three patients were lost to follow-up).

\section{Discussion}

In this study, the changes in $\mathrm{StO}_{2}, \mathrm{BVF}$, and perfusion of PWS were measured before and during V-PDT with the intravenous injection of HiPorfin for the first time. A wide variety of responses were noted in different lesions of different patients and even in different regions within the same lesion, but the general trend has demonstrated an increase in the localized mean $\mathrm{StO}_{2}, \mathrm{BVF}$, and skin perfusion after the initiation of $\mathrm{V}-\mathrm{PDT}$. $\mathrm{StO}_{2}$ increased first and then fluctuated. The overall trend of BVF change in PWS was similar to that of perfusion change. The BVF and the perfusion of PWS lesions increased first and then slowly 
A

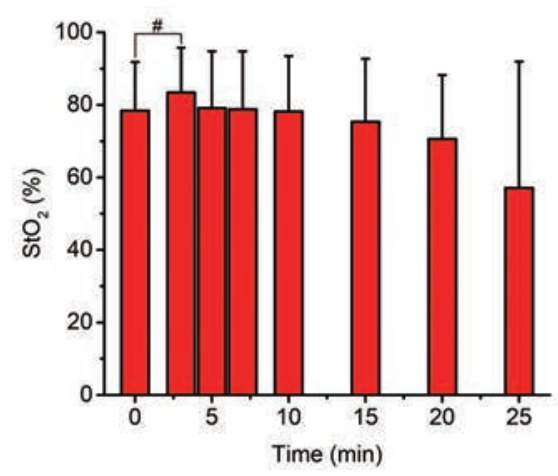

B

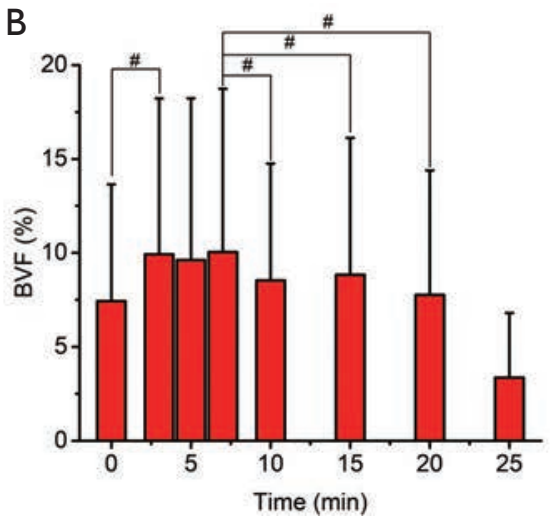

C

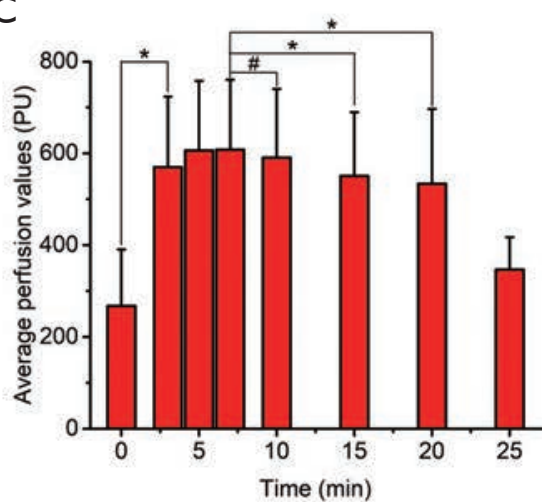

Figure 4 Changes of (A) $\mathrm{StO}_{2}$, (B) BVF and (C) perfusion in the PWS lesions of all patients during V-PDT. The error bars represent the $\mathrm{SD}$ of the data. *, $\mathrm{P}<0.001$;,, $\mathrm{P}<0.05$. PWS, port-wine stains; $\mathrm{StO}_{2}$, oxygen saturation; $\mathrm{BVF}$, blood volume fraction.

decreased to a relatively low-level during V-PDT.

Before V-PDT, majority of the PWS lesions have higher levels of blood perfusion and BVF than the contralateral control sites, and the differences between the PWS lesions and the contralateral control sites may vary from patient to patient. However, no significant difference was observed in the $\mathrm{StO}_{2}$ of the PWS lesions and the contralateral control sites. Given that PWS lesions contain abnormally dilated blood vessels, the high levels of blood perfusion and BVF in PWS lesions could be due to the increased blood volume of the ectatic vessels (29). The differences between the blood perfusion values in the PWS lesions and in the contralateral control sites may vary remarkably. Even for the same PWS lesion with same skin color, a considerable spatial heterogeneity of blood perfusion was observed across the skin surface, which may be ascribed to the heterogeneous vascular malformations in PWS. The 3-dimensional reconstruction of the PWS vascular anatomy based on the serial histological sections has revealed the multiple clusters of small diameter blood vessels and nonuniform distribution of blood vessels (30). Furthermore, the thickness and color of PWS lesions vary substantially from one patient to another or even from lesion to lesion on the same patient $(19,30)$. Consequently, a large inter- and intra-patient heterogeneity of microvasculature in the PWS lesion were observed and may cause different responses to the V-PDT. Furthermore, the cutaneous $\mathrm{StO}_{2}, \mathrm{BVF}$, and perfusion may be influenced by several factors, such as physical activity (31) and ambient temperature (32). In order to minimize the external influence, all measurements were performed with the subject in a supine position at a temperature-controlled room, and all the participants had an acclimatization period before measurement and were asked to remain still during measurements.

According to previous studies, the most prominent photodynamic microvascular damage may occur 0 to $30 \mathrm{~min}$ after intravenous administration $(8,9)$. Therefore, light irradiation was performed immediately after injection and irradiation times ranging between 15-25 min were used in this study. Most patients have varying degrees of fading in this study, indicating that the V-PDT is effective for the treatment of PWS. The effectiveness of V-PDT is highly dependent on the photosensitizer concentration and the oxygen supply within the target vasculatures, which may be influenced by the blood perfusion particularly for the intravenous injection of photosensitizer (26). The skin perfusion and oxygen supply within the target vasculature would change caused by the vascular damage during V-PDT, and monitoring blood perfusion and oxygenation in PWS during V-PDT may provide a deeper understanding of the responses of microcirculation and oxygenation in PWS lesions to V-PDT.

As with our previous study, during V-PDT, the perfusion in PWS significantly increased after the initiation of treatment, reached a peak within $10 \mathrm{~min}$, and then decreased gradually (15). Similar to perfusion change, the BVF change increased first and then decreased. The perfusion measured using LDI is proportional to the product of the average speed of the blood cells and their number concentration, which is often referred to as blood volume (33). Therefore, BVF has a similar variation tendency to the perfusion of PWS in response to V-PDT. The possible explanation for blood perfusion changes during V-PDT has been explored previously (15). The initial increase in perfusion may be 
Table 2 The changes in $\mathrm{StO}_{2}$, BVF and perfusion in PWS lesions during V-PDT

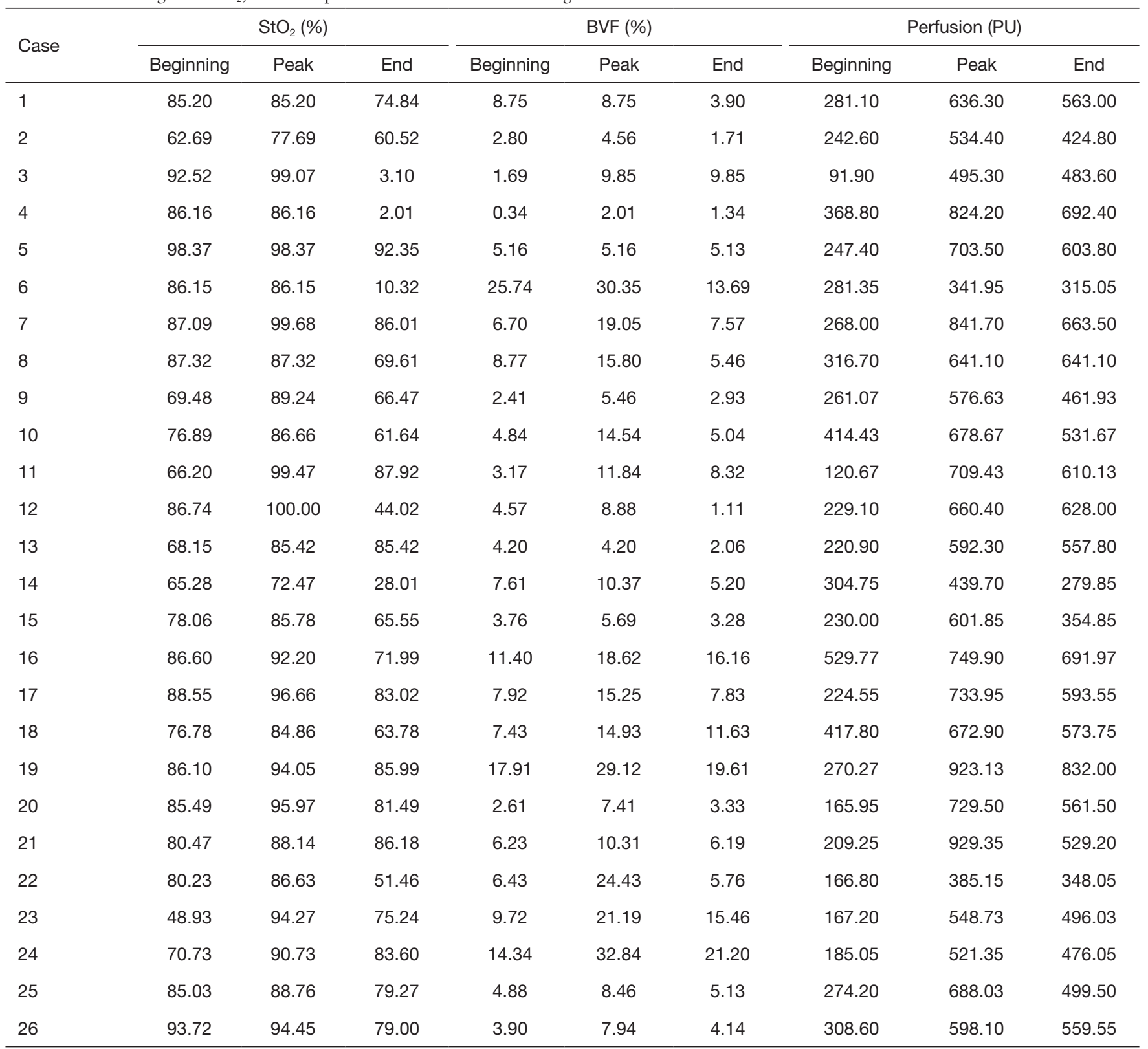

PWS, port-wine stains; V-PDT, vascular targeted photodynamic therapy; BVF, blood volume fraction; StO $_{2}$, oxygen saturation.

attributed to the laser irradiation-induced local thermal hyperemia (34). The infrared thermometer revealed that the temperature of the PWS lesions being treated increased to about $39-42{ }^{\circ} \mathrm{C}$ during V-PDT, and the local heating of the skin by laser irradiation lead to a direct, substantial and localized vasodilation in the area being heated (35). The decrease in perfusion might be attributed to the malformed blood vessels damage caused by photodynamic reaction.
Previous study has shown that the endothelial cell damage caused by photodynamic reaction can lead to the formation of thrombosis within the vessel lumen, which triggers a serial physiological cascade of responses including platelet aggregation, release of vasoactive molecules, leukocyte adhesion, increased vascular permeability, and vessel constriction (36). Moreover, the sequence of vascular events may persist for a long time after the V-PDT treatment (18), 
and mild immune response might be evoked. Furthermore, we previously showed a persistent V-PDT effect, leading to the closure of the majority of microvasculature in tumor after Verteporfin-mediated V-PDT treatment (37). This study further confirm that the targeted vessels cannot be completely shut down right after PDT, and the PDT effects may last for some time after the V-PDT treatment. The large error bar of the changes in blood perfusion and BVF has shown the significant variations in the vascular response to V-PDT from site to site within the same treated lesion, which may be attributed to heterogeneous vascular malformations in PWS lesions (30).

The $\mathrm{StO}_{2}$ [or the partial pressure of oxygen $\left(\mathrm{pO}_{2}\right)$ ] in animal models before, during, and after PDT has been monitored in several studies, and the results show significant changes in oxygenation during and after PDT but with different patterns of responses (38-41). A rapid decline in $\mathrm{pO}_{2}$ was observed immediately after commencing ALAPDT in mouse and rat models $(38,39)$, whereas other studies have demonstrated large increases in tissue oxygenation during ALA-PDT (40). The variability can be attributed to differences in animal models, treatment protocols (topical application versus i.v. injection), applied photosensitizers, and dosimetry. A clinical observation has shown that a significant reduction in the mean blood $\mathrm{StO}_{2}$ occurs maximally in the first half of treatment during topical Methylaminolaevulinate (MAL)-PDT by using optical reflectance spectroscopy (42). Different from the topical PDT for the treatment of cancer diseases, our results showed that $\mathrm{StO}_{2}$ has increased slightly first and then fluctuated in PWS lesions during V-PDT after the systematic administration of HiPorfin. The increase can be attributed to an increase in blood perfusion, thereby increasing the supply rate of oxygen. The subsequent fluctuation in $\mathrm{StO}_{2}$ may be due to the photodynamic consumption and/or vessel occlusion. The photodynamic reaction-induced oxygen changes are transient and occur during the time of laser irradiation. Moreover, depending on the cause, vessel occlusion and stasis can be either transient or permanent (40).

In this study, the changes in the $\mathrm{StO}_{2}$ and perfusion that occurred in PWS lesions during V-PDT in vivo were monitored simultaneously for the first time. Both of these optical technologies (LDI and DRS) provided detailed insights into the vascular responses (including oxygenation and perfusion) to V-PDT treatment of PWS and facilitated our understanding of the basic physiological mechanisms during V-PDT. It is found that the $\mathrm{StO}_{2}$ increased first and then fluctuated during V-PDT, indicating that no significant depletion of oxygen was observed during V-PDT treatment of PWS. According to this finding, oxygen would not be the major limiting factor for efficacy of V-PDT treatment of PWS. The BVF and the perfusion of PWS lesions increased after the initiation of V-PDT, and then gradually decreased. The decrease in perfusion might be ascribed to the malformed blood vessels damage caused by photodynamic reaction. Therefore, in clinical practice, LDI was suitable for monitoring the vascular response to V-PDT treatment of PWS.

This study is limited by the number of patients with clinical time constraints. However, we have demonstrated that both the DRS and LDI can be successfully used in the standard clinical setting for V-PDT monitoring and investigation. Substantial inter- and intra-patient heterogeneity of responses of PWS lesions to V-PDT were observed. No marked correlation between the degree of changes in the blood perfusion or $\mathrm{StO}_{2}$ during V-PDT and the color bleaching rates of PWS was observed in the investigation. Nevertheless, we cannot conclude that there is no correlation between them, because patient-specific factors such as: gender, age, general health, smoking history, and types of PWS, has not been controlled. Future work should monitor significantly more patients to confirm the observations within the patients of this cohort and extend the investigation for PDT optimization.

\section{Conclusions}

In summary, the LDI and the DRS detection systems were successfully applied to noninvasively assess the changes in $\mathrm{StO}_{2}$, BVF, and perfusion that occurred in the PWS lesions during V-PDT in vivo with the systematic administration of the porphyrin-based photosensitizer HiPorfin. V-PDT is an effective therapeutic modality in treating PWS. The changes in $\mathrm{StO}_{2}, \mathrm{BVF}$, and perfusion of PWS lesions were monitored before and during V-PDT, and the common trends for each parameter were identified. These methods can also be useful in facilitating our understanding of the basic physiological mechanisms during V-PDT and have the potential to guide clinical V-PDT.

\section{Acknowledgments}

The authors would acknowledge Zhihai Qiu for the help of data fitting. The authors acknowledge DRS system calibration work supported by Beijing Institute of Technology Analysis \& Testing Center. 
Funding: This study was supported by the National Natural Science Foundation of China (No. 61635014, 61527827, 61835015), the National Key R\&D Program of China (2019YFC0119300), CAMS Innovation Fund for Medical Sciences (CIFMS) (2019-I2M-5-061), and Beijing Institute of Technology Research Fund Program for Young Scholars.

\section{Footnote}

Reporting Checklist: The authors have completed the STROBE reporting checklist. Available at http://dx.doi. org/10.21037/atm-20-3210

Data Sharing Statement: Available at http://dx.doi. org/10.21037/atm-20-3210

Conflicts of Interest: All authors have completed the ICMJE uniform disclosure form (available at http://dx.doi. org/10.21037/atm-20-3210). The authors have no conflicts of interest to declare.

Ethical Statement: The authors are accountable for all aspects of the work in ensuring that questions related to the accuracy or integrity of any part of the work are appropriately investigated and resolved. All procedures performed in this study involving human participants were in accordance with the Declaration of Helsinki (as revised in 2013). The study was approved by the Ethic Committee of Chinese PLA General Hospital (No. 20100404), and informed consent was taken from all the patients (or guardians) prior to the experiments.

Open Access Statement: This is an Open Access article distributed in accordance with the Creative Commons Attribution-NonCommercial-NoDerivs 4.0 International License (CC BY-NC-ND 4.0), which permits the noncommercial replication and distribution of the article with the strict proviso that no changes or edits are made and the original work is properly cited (including links to both the formal publication through the relevant DOI and the license). See: https://creativecommons.org/licenses/by-nc-nd/4.0/.

\section{References}

1. Alper JC, Holmes LB. The incidence and significance of birthmarks in a cohort of 4,641 newborns. Pediatr Dermatol 1983;1:58-68.

2. Kelly KM, Choi B, McFarlane S, et al. Description and analysis of treatments for port-wine stain birthmarks. Arch Facial Plast Surg 2005;7:287-94.

3. Hagen SL, Grey KR, Korta DZ, et al. Quality of life in adults with facial port-wine stains. J Am Acad Dermatol 2017;76:695-702.

4. Aguilar G, Choi B, Broekgaarden M, et al. An overview of three promising mechanical, optical, and biochemical engineering approaches to improve selective photothermolysis of refractory port wine stains. Ann Biomed Eng 2012;40:486-506.

5. Lanigan SW. Port-wine stains unresponsive to pulsed dye laser: explanations and solutions. Br J Dermatol 1998;139:173-7.

6. Savas JA, Ledon JA, Franca K, et al. Pulsed dye laserresistant port-wine stains: mechanisms of resistance and implications for treatment. Br J Dermatol 2013;168:941-53.

7. Lipner SR. Topical adjuncts to pulsed dye laser for treatment of port wine stains: review of the literature. Dermatol Surg 2018;44:796-802.

8. Gu Y, Huang NY, Liang J, et al. Clinical study of 1949 cases of port wine stains treated with vascular photodynamic therapy (Gu's PDT). Ann Dermatol Venereol 2007;134:241-4.

9. Qiu H, Gu Y, Wang Y, et al. Twenty years of clinical experience with a new modality of vascular-targeted photodynamic therapy for port wine stains. Dermatol Surg 2011;37:1603-10.

10. Zhao Y, Tu P, Zhou G, et al. Hemoporfin photodynamic therapy for port-wine stain: a randomized controlled trial. PLoS One 2016;11:e0156219.

11. Ma G, Han Y, Ying H, et al. Comparison of two generation photosensitizers of PsD-007 and hematoporphyrin monomethyl ether photodynamic therapy for treatment of port-wine stain: a retrospective study. Photobiomodul Photomed Laser Surg 2019;37:376-80.

12. Gao K, Huang $Z$, Yuan KH, et al. Side-by-side comparison of photodynamic therapy and pulseddye laser treatment of port-wine stain birthmarks. Br J Dermatol 2013;168:1040-6.

13. Zhang B, Zhang TH, Huang Z, et al. Comparison of pulsed dye laser (PDL) and photodynamic therapy (PDT) for treatment of facial port-wine stain (PWS) birthmarks in pediatric patients. Photodiagnosis Photodyn Ther 2014;11:491-7.

14. Ren J, Li P, Zhao H, et al. Assessment of tissue perfusion changes in port wine stains after vascular targeted photodynamic therapy: a short-term follow-up study. Lasers Med Sci 2014;29:781-8. 
15. Chen D, Ren J, Wang Y, et al. Intraoperative monitoring of blood perfusion in port wine stains by laser Doppler imaging during vascular targeted photodynamic therapy: A preliminary study. Photodiagnosis Photodyn Ther 2016;14:142-51.

16. Sharif SA, Taydas E, Mazhar A, et al. Noninvasive clinical assessment of port-wine stain birthmarks using current and future optical imaging technology: a review. Br J Dermatol 2012;167:1215-23.

17. Chen D, Ren J, Wang Y, et al. Relationship between the blood perfusion values determined by laser speckle imaging and laser Doppler imaging in normal skin and port wine stains. Photodiagnosis Photodyn Ther 2016;13:1-9.

18. Qiu H, Zhou Y, Gu Y, et al. Monitoring microcirculation changes in port wine stains during vascular targeted photodynamic therapy by laser speckle imaging. Photochem Photobiol 2012;88:978-84.

19. Zhao S, Gu Y, Xue P, et al. Imaging port wine stains by fiber optical coherence tomography. J Biomed Opt 2010;15:036020.

20. Christman MP, Feng H, Holmes J, et al. Treating port wine stain birthmarks using dynamic optical coherence tomography-guided settings. J Am Acad Dermatol 2019. [Epub ahead of print].

21. Xu D, Yang S, Wang Y, et al. Noninvasive and highresolving photoacoustic dermoscopy of human skin. Biomed Opt Express 2016;7:2095-102.

22. Mazhar A, Sharif SA, Cuccia JD, et al. Spatial frequency domain imaging of port wine stain biochemical composition in response to laser therapy: A pilot study. Lasers Surg Med 2012;44:611-21.

23. Wang Y, Gu Y, Liao X, et al. Fluorescence monitoring of a photosensitizer and prediction of the therapeutic effect of photodynamic therapy for port wine stains. Exp Biol Med (Maywood) 2010;235:175-80.

24. Qiu Z, Chen D, Wang Y, et al. Monitoring blood volume fraction and oxygen saturation in port-wine stains during vascular targeted photodynamic therapy with diffuse reflectance spectroscopy: Results of a preliminary case study. Photonics Lasers Med 2014;3:273-80.

25. Qiu Z, Yao G, Chen D, et al. Determination of optical and microvascular parameters of port wine stains using diffuse reflectance spectroscopy. Adv Exp Med Biol 2016;923:359-65.

26. Becker TL, Paquette AD, Keymel KR, et al. Monitoring blood flow responses during topical ALA-PDT. Biomed Opt Express 2010;2:123-30.

27. Daly SM, Leahy MJ. 'Go with the flow': A review of methods and advancements in blood flow imaging. J Biophotonics 2013;6:217-55.

28. Kim A, Roy M, Dadani F, et al. A fiberoptic reflectance probe with multiple source-collector separations to increase the dynamic range of derived tissue optical absorption and scattering coefficients. Optics Express 2010;18:5580-94.

29. Barsky SH, Rosen S, Geer DE, et al. The nature and evolution of port wine stains: a computer-assisted study. J Invest Dermatol 1980;74:154-7.

30. Smithies DJ, vanGemert MJC, Hansen MK, et al. Threedimensional reconstruction of port wine stain vascular anatomy from serial histological sections. Phys Med Biol 1997;42:1843-7.

31. Johnson JM. Exercise and the cutaneous circulation. Exerc Sport Sci Rev 1992;20:59-97.

32. McGill DJ, Mackay IR. The effect of ambient temperature on capillary vascular malformations. Br J Dermatol 2006;154:896-903.

33. Leahy MJ, Enfield JG, Clancy NT, et al. Biophotonic methods in microcirculation imaging. Medical Laser Application 2007;22:105-26.

34. Roustit M, Cracowski JL. Non-invasive assessment of skin microvascular function in humans: an insight into methods. Microcirculation 2012;19:47-64.

35. Charkoudian N. Skin blood flow in adult human thermoregulation: How it works, when it does not, and why. Mayo Clinic Proceedings 2003;78:603-12.

36. Krammer B. Vascular effects of photodynamic therapy. Anticancer Res 2001;21:4271-7.

37. Chen D, Yuan W, Park H-C, et al. In vivo assessment of vascular-targeted photodynamic therapy effects on tumor microvasculature using ultrahigh-resolution functional optical coherence tomography. Biomedical Optics Express 2020;11:4316-25.

38. Sitnik TM, Hampton JA, Henderson BW. Reduction of tumour oxygenation during and after photodynamic therapy in vivo: effects of fluence rate. Br J Cancer 1998;77:1386-94.

39. Curnow A, Haller JC, Bown SG. Oxygen monitoring during 5-aminolaevulinic acid induced photodynamic therapy in normal rat colon. Comparison of continuous and fractionated light regimes. J Photochem Photobiol B 2000;58:149-55.

40. Pogue BW, O'Hara JA, Goodwin IA, et al. Tumor Po2 changes during photodynamic therapy depend upon photosensitizer type and time after injection. Comp Biochem Physiol A Mol Integr Physiol 2002;132:177-84. 
41. Neuschmelting V, Kim K, Malekzadeh-Najafabadi J, et al. WST11 vascular targeted photodynamic therapy effect monitoring by multispectral optoacoustic tomography (MSOT) in mice. Theranostics 2018;8:723-34.

Cite this article as: Chen D, Wang Y, Zhao H, Qiu H, Wang Y, Yang J, Gu Y. Monitoring perfusion and oxygen saturation in port-wine stains during vascular targeted photodynamic therapy. Ann Transl Med 2021;9(3):214. doi: 10.21037/atm-203210
42. Tyrrell J, Thorn C, Shore A, et al. Oxygen saturation and perfusion changes during dermatological methylaminolaevulinate photodynamic therapy. Br J Dermatol 2011;165:1323-31. 